Cite this: Phys. Chem. Chem. Phys., 2011, 13, 3630-3633

\title{
Saturation factor of nitroxide radicals in liquid DNP by pulsed ELDOR experiments
}

\author{
Maria-Teresa Türke and Marina Bennati* \\ Received 12th October 2010, Accepted 4th January 2011 \\ DOI: $10.1039 / \mathrm{c0cp02126a}$
}

We propose the use of the pulse electron double resonance (ELDOR) method to determine the effective saturation factor of nitroxide radicals for dynamic nuclear polarization (DNP) experiments in liquids. The obtained values for the nitroxide radical TEMPONE-D, ${ }^{15} \mathrm{~N}$ at different concentrations are rationalized in terms of spin relaxation and are shown to fulfil the Overhauser theory.

Dynamic nuclear polarization (DNP) in aqueous solution at ambient conditions is a major topic of current efforts to enhance the sensitivity of high resolution NMR and magnetic resonance imaging. ${ }^{1-3}$ In the liquid state, DNP is governed by the Overhauser mechanism, ${ }^{4,5}$ in which polarization is transferred from paramagnetic centers to coupled nuclear spins by microwave pumping of the EPR line. The enhancements $\varepsilon$ depend on four factors, i.e. the ratio of the gyromagnetic constants of the electron spin $\gamma_{\mathrm{s}}$ and the target nucleus $\gamma_{\mathrm{I}}$, the coupling factor $\xi$, the leakage factor $f$ and the effective saturation factor $s_{\text {eff }}$ of the EPR line: ${ }^{6}$

$$
\varepsilon=1-s_{\mathrm{eff}} f \xi\left|\gamma_{\mathrm{s}}\right| / \gamma_{\mathrm{I}}
$$

In recent studies, nitroxide radicals have been favoured as polarizing agents for DNP since they are soluble in water, well compatible with biological systems, non-toxic and have been found to account for large DNP enhancements at magnetic fields up to $9 \mathrm{~T}^{7,8}$ However, the determination of the saturation factor for this class of polarizers has emerged as one of the major difficulties in rationalizing the observed DNP enhancements in terms of the Overhauser equation (1). ${ }^{8-12}$ Due to the strong hyperfine coupling between the electron and the nitrogen nucleus, which splits the EPR spectrum into two $\left({ }^{15} \mathrm{~N}\right)$ or three $\left({ }^{14} \mathrm{~N}\right)$ separate lines, the saturation factor, defined as $s_{\text {eff }}=\left(S^{\mathrm{B}}-\left\langle S_{z}\right\rangle\right) / S^{\mathrm{B}}$ with $S^{\mathrm{B}}$ the Boltzmann polarization of the electron spin, cannot be simply extracted from the saturation behaviour of the pumped line according to the Bloch equation, as formerly suggested for trityl radicals. ${ }^{13}$ Moreover, the expectation value of the electron spin polarization $\left\langle S_{z}\right\rangle$ depends on the population of all energy levels involved. In recent years, this issue has been debated in the literature and several theoretical models

were proposed to calculate the saturation factor for nitroxides $^{9-11}$ but to date no direct experimental approach has been provided.

In this paper, we show that the effective saturation factor in Overhauser DNP can be determined by a pulsed electron double resonance (ELDOR) experiment, ${ }^{14}$ which measures the intensity of a hyperfine line when pumping a coupled hyperfine line. This approach is demonstrated with a ${ }^{15} \mathrm{~N}$ containing nitroxide because this gives the maximum enhancement for DNP and is relevant for future DNP applications. However, it is fully applicable to radicals incorporating a ${ }^{14} \mathrm{~N}$ nucleus as well. Our experiments were performed at X-band (9.7 GHz electron Larmor frequency) in a commercial dielectric EPR/ENDOR resonator (Bruker EN4118X-MD4), as used for DNP, ${ }^{12}$ that is overcoupled to permit irradiation at two frequencies with $\Delta \nu$ up to $100 \mathrm{MHz}$. The saturation factors were measured for ${ }^{15} \mathrm{~N}$ - and ${ }^{2} \mathrm{H}$-labelled TEMPONE (4-Oxo-TEMPO) dissolved in water in concentrations ranging from 0.5 to $25 \mathrm{mM}$. These values were compared to theoretical predictions based on the analytical solution of the rate equations for the electron spin coupled to the ${ }^{15} \mathrm{~N}$ nucleus. Finally, the saturation factors allow extraction of the coupling factors for DNP according to eqn (1).

To discuss the physical parameters that determine the saturation factor, we illustrate in Fig. 1 the four energy levels of an electron spin $\left(S=\frac{1}{2}\right)$ strongly coupled to a nucleus with spin $I=\frac{1}{2}$ in a static magnetic field as for a ${ }^{15} \mathrm{~N}$ labelled nitroxide radical. The expectation value of the electron spin

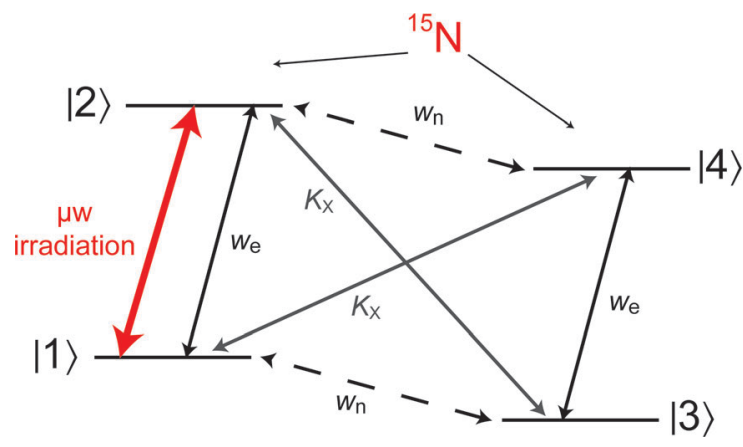

Fig. 1 Relaxation scheme for an $S=\frac{1}{2}$ and $I=\frac{1}{2}$ coupled system containing the spin lattice relaxation rates $w_{\mathrm{e}}, w_{\mathrm{n}}$ and the spin exchange rate $K_{x}$. Continuous microwave irradiation on one of the EPR transitions as applied during the DNP experiment is indicated. 
polarization $\left\langle S_{z}\right\rangle$ entering the saturation factor depends on the populations (per unit volume) $N_{i}$ of the four energy levels:

$$
\left\langle\hat{S}_{z}\right\rangle=\sum_{i}\left\langle i\left|\hat{S}_{z}\right| i\right\rangle \cdot N_{i}=\frac{1}{2}\left(N_{2}-N_{1}+N_{4}-N_{3}\right)
$$

The relevant relaxation rates in solution ${ }^{15}$ are the electron spin lattice relaxation rate $w_{\mathrm{e}}$, the nitrogen nuclear spin lattice relaxation rate $w_{\mathrm{n}}$ and the Heisenberg spin exchange rate (per concentration) $K_{x}$.

The observable EPR signals $i_{1,2}$ of the two lines can be described in terms of the differences in the populations $N_{1,2,3,4}$ :

$$
\begin{aligned}
& i_{1}=\left(N_{1}-N_{2}\right)-\left(N_{1}^{\mathrm{B}}-N_{2}^{\mathrm{B}}\right) \\
& i_{2}=\left(N_{3}-N_{4}\right)-\left(N_{3}^{\mathrm{B}}-N_{4}^{\mathrm{B}}\right)
\end{aligned}
$$

Within this definition, the signals at thermal equilibrium $\left(N_{1}^{\mathrm{B}}-N_{2}^{\mathrm{B}}\right)$ and $\left(N_{3}^{\mathrm{B}}-N_{4}^{\mathrm{B}}\right)$ are subtracted. ${ }^{15}$ The time evolution of the signal intensities is determined by the competing relaxation rates as summarized in the following coupled rate equations:

$$
\begin{aligned}
& \frac{\mathrm{d} i_{1}}{\mathrm{~d} t}=-\left(2 w_{\mathrm{e}}+w_{\mathrm{n}}+\frac{N}{2} K_{x}\right) i_{1}+\left(w_{\mathrm{n}}+\frac{N}{2} K_{x}\right) i_{2} \\
& \frac{\mathrm{d} i_{2}}{\mathrm{~d} t}=-\left(2 w_{\mathrm{e}}+w_{\mathrm{n}}+\frac{N}{2} K_{x}\right) i_{2}+\left(w_{\mathrm{n}}+\frac{N}{2} K_{x}\right) i_{1}
\end{aligned}
$$

with the total population $N=N_{1}+N_{2}+N_{3}+N_{4}$. Their solution is readily obtained as a sum of two exponential functions containing combinations of the relaxation rates: ${ }^{15}$

$$
i_{1,2}=A_{1,2} \mathrm{e}^{-2 w_{\mathrm{e}} t}+B_{1,2} \mathrm{e}^{-\left(2 w_{\mathrm{e}}+2 w_{\mathrm{n}}+N K_{x}\right) t}
$$

The prefactors $A_{1,2}$ and $B_{1,2}$ are given by the initial conditions.

In the present work, we consider that during the DNP experiment the $\mathrm{cw}$ microwave irradiation in resonance with one of the EPR transitions (for instance $|1\rangle \leftrightarrow|2\rangle$ ) generates a steady state of all populations. Therefore, the rates in eqn (4) drop to zero. Additionally, if the irradiation is such that it achieves saturation of $|1\rangle \leftrightarrow|2\rangle$, i.e. $N_{1}=N_{2}$, then $i_{1}=$ $-\left(N_{1}^{\mathrm{B}}-N_{2}^{\mathrm{B}}\right)$. Assuming $N_{1}^{\mathrm{B}}-N_{2}^{\mathrm{B}}=N_{3}^{\mathrm{B}}-N_{4}^{\mathrm{B}}$, we derive the saturation level $s_{2}$ of the second EPR transition $|3\rangle \leftrightarrow|4\rangle$ as:

$$
s_{2}=\frac{\left(N_{3}^{\mathrm{B}}-N_{4}^{\mathrm{B}}\right)-\left(N_{3}-N_{4}\right)}{\left(N_{3}^{\mathrm{B}}-N_{4}^{\mathrm{B}}\right)}=1-\frac{2}{2+\frac{w_{\mathrm{n}}}{w_{\mathrm{e}}}+\frac{N K_{x}}{2 w_{\mathrm{e}}}}
$$

This expression was also reported by Hyde et al. ${ }^{14}$ as a limiting case within the general theory of saturation transfer and double resonance effects in EPR. ${ }^{16}$ However, the relaxation rates entering eqn (6) are unknown and can be determined only in an electron double resonance experiment. ${ }^{14,17}$ Furthermore, this type of experiment opens up the possibility to directly monitor the saturation level of each coupled hyperfine line.

Fig. 2 shows the normalized intensity of the electron FID (free induction decay) detected on the high-field hyperfine line after baseline subtraction as a function of the ELDOR frequency, on which a saturating pulse of $1 \mu$ s length was applied. The 12 ns detection pulse with an excitation bandwidth of about $80 \mathrm{MHz}$ was set specifically $30 \mathrm{MHz}$ off-resonance to the high field side of the line to avoid simultaneous excitation

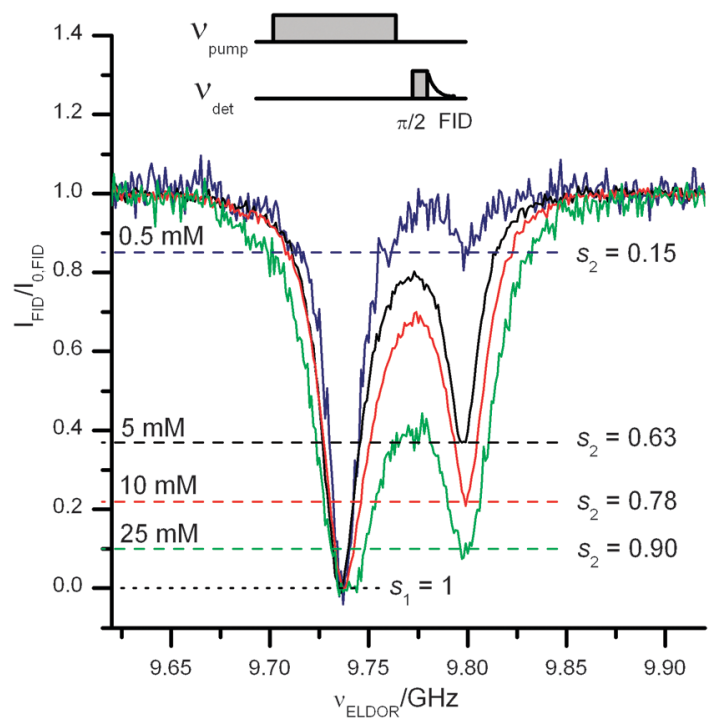

Fig. 2 Normalized EPR FID intensity of the hyperfine line at $9.74 \mathrm{GHz}$ as a function of the frequency of the saturating pulse and for different concentrations of TEMPONE-D,${ }^{15} \mathrm{~N}$ in water at room temperature.

of the low-field transition, which occurs $60 \mathrm{MHz}$ below the high-field line. With the overcoupled dielectric cavity, the background ringing was reduced to an extent that allowed the detection of the short EPR FID at room temperature.

Fig. 2 illustrates that the FID intensity is lowered down to the background ringing of the cavity, as soon as the saturating pulse comes into resonance with the detection frequency. This indicates that saturation of the pumped line is indeed achieved $\left(s_{1}=1\right)-$ the precondition for eqn (6). The error due to the ringing amounts to less than $\sim 10 \%$ of the total line intensity. Our previous studies on the saturation level of one line ${ }^{12}$ also confirm that the microwave $B_{1}$ field in the overcoupled dielectric resonator is sufficient for saturation of the pumped line, i.e. $s_{1} \geq 0.9$ at $B_{1}=2 \mathrm{G}$ for concentrations $\leq 25 \mathrm{mM}$. When the saturating pulse is resonant with the other (low-field) EPR transition, we observe another drop in the FID intensity (line at $9.8 \mathrm{GHz}$ in Fig. 2), which depends on the concentration of the radical. The reduction of the normalized signal directly reflects the saturation level of the second line $s_{2}$ (eqn (6)) that is achieved by the ELDOR effect. These values are indicated in Fig. 2.

The effective DNP saturation factor is then obtained as the average of the saturation levels of the two EPR transitions, $\dagger$ i.e.

$$
s_{\text {eff }}=\left(s_{1}+s_{2}\right) / 2
$$

It should be noted that the condition $s_{1}=1$ is required for the analytical solution of $s_{2}$ based on eqn (5) and (6), but the direct experimental determination of the saturation factors $s_{1}$ and $s_{2}$ is independent of this and is valid for any available microwave power.

The results displayed in Fig. 2 show that saturation transfer for the ${ }^{15} \mathrm{~N}$ labelled nitroxide radical is strongly dependent on the concentration, i.e. almost no effect is observed at $0.5 \mathrm{mM}$ whereas both transitions are nearly completely saturated at $25 \mathrm{mM}$. Therefore, saturation factors close to one in 
Overhauser DNP can be achieved with ${ }^{15} \mathrm{~N}$ labelled nitroxide radicals, although only one of the hyperfine lines is irradiated, if high radical concentrations ( $\geq 25 \mathrm{mM}$ ) are used. We note that the results for 5 and $10 \mathrm{mM}$ agree well with the ones of our initial publication. ${ }^{12}$ Additionally, the observed concentration dependence indicates that at room temperature the intramolecular nitrogen nuclear relaxation rate $w_{\mathrm{n}}$ is small and that Heisenberg spin exchange dominates saturation transfer of nitroxides at the relevant concentrations for DNP ( $\geq 5 \mathrm{mM}$ ). In this case eqn (6) can be approximated as:

$$
s_{2} \approx 1-\frac{2}{2+N K_{x} /\left(2 w_{\mathrm{e}}\right)}
$$

This expression could be used to check our experimental results against the theory, however the relaxation rates entering (8) $w_{\mathrm{e}}$ and $w_{x}=N K_{x}$ had to be first measured independently using a combined polarization recovery (PR) EPR and ELDOR experiment, as described below. ${ }^{15,17,18}$

The experiment was carried out with a $5 \mathrm{mM}$ sample of TEMPONE-D, ${ }^{15}$ N. Again, the EPR FID was detected on the high field line and an initial high-turning angle pulse of $100 \mathrm{~ns}$ length was applied either on the same (PR-EPR) or the other (PR-ELDOR) hyperfine line. The time interval between the two pulses was incremented to record the recovery of the FID back to thermal equilibrium (see Fig. 3). The form of the

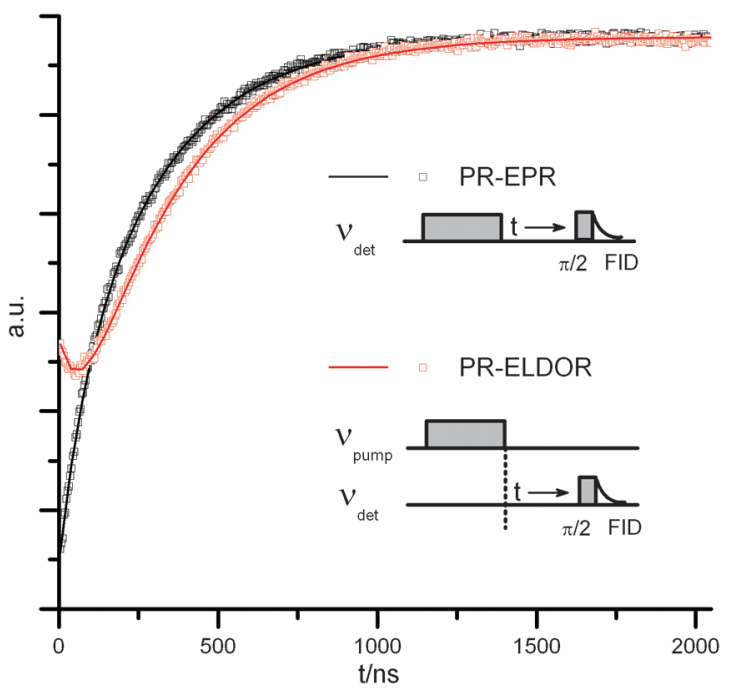

Fig. 3 Recovery curves of the FID in EPR and ELDOR polarization recovery measurements on $5 \mathrm{mM}$ TEMPONE-D, ${ }^{15} \mathrm{~N}$ in water. Both curves were fitted (solid lines) to (5) with shared time constants. The inset shows the respective pulse sequences. recovery curve in Fig. 3 of the PR-ELDOR experiment clearly illustrates the counteracting mechanisms of polarization transfer and recovery to the Boltzmann state. Both curves were fitted to the time dependent solution (5) with shared parameters $T_{\mathrm{le}}=\left(2 w_{\mathrm{e}}\right)^{-1}$ and $T_{\mathrm{b}}=\left(2 w_{\mathrm{e}}+2 w_{\mathrm{n}}+w_{x}\right)^{-1}$. Under the assumption that $w_{\mathrm{n}}$ is negligible, the fit contains only two unknowns ( $w_{x}$ and $w_{\mathrm{e}}$ ), which can be extracted from the two recovery curves at a single concentration value yielding the electron spin lattice relaxation rate $T_{1 \mathrm{e}}=298 \mathrm{~ns}$ and $T_{\mathrm{b}}=76 \mathrm{~ns}$. We obtain $w_{x}=2.0 \times 10^{9} \mathrm{~s}^{-1}$ for $1 \mathrm{M}$ concentration. Both relaxation parameters are in good agreement with the literature values. ${ }^{19,20}$

As a check for the assumption used above (negligible $w_{\mathrm{n}}$ at concentrations $\geq 5 \mathrm{mM}$ ) we have recorded similar curves for the $0.5 \mathrm{mM}$ concentrated sample (data not shown) and we obtain $\left(2 w_{\mathrm{n}}+w_{x}\right)^{-1} \geq 1 \mu \mathrm{s}$. This indicates that $1 /\left(2 w_{\mathrm{n}}\right) \geq 1 \mu \mathrm{s}$ and the assumption is valid.

In Table 1 we compare the saturation levels $s_{2}$, calculated using expression (8) and the rates determined by the polarization recovery experiment, with the saturation levels directly observed in the ELDOR experiment of Fig. 2. They are in very good agreement for all concentrations, which confirms the validity of our approach.

The measured effective saturation factors could be used to evaluate the Overhauser eqn (1) for the observed DNP enhancements at $9.7 \mathrm{GHz}$. With a previously reported DNP setup at $0.35 \mathrm{~T}\left(9.7 \mathrm{GHz} \text { EPR, } 15 \mathrm{MHz}{ }^{1} \mathrm{H} \mathrm{NMR}\right)^{12}$ we have experimentally determined the maximum achievable DNP enhancement factors of the water ${ }^{1} \mathrm{H}$ signal at $\sim 20 \mathrm{~W}$ microwave power. This power is higher than that employed in the previous paper $^{12}(4 \mathrm{~W})$ and leads to clear saturation of the DNP enhancements at all considered concentrations as displayed in Fig. 4. For this measurement, the sample dimensions were optimized $(0.45 \mathrm{~mm}$ ID, $3 \mathrm{~mm}$ length) as to avoid microwave heating of the sample. ${ }^{12}$ The maximum enhancement factors are reported in Table 1. Using these values for $\varepsilon$, the effective saturation factors $s_{\text {eff }}$ and the leakage factors $f$ from ref. 21 , we have evaluated the coupling factor $\xi$ from the Overhauser equation (1), as summarized in Table 1. The coupling factor calculated from the experiments at the three different concentrations amounts to $0.33 \pm 0.02$, and is independent of concentration as expected. This value is in agreement with the coupling factors derived by nuclear magnetic relaxation dispersion $(0.33-0.35)^{21}$ and with molecular dynamics simulations $(0.30){ }^{22}$

We conclude that pulsed ELDOR provides the suitable approach to evaluate the saturation factors for DNP in liquid solution. The individual saturation levels of separate hyperfine

Table 1 Saturation factors determined by pulsed ELDOR and evaluation of Overhauser DNP

\begin{tabular}{|c|c|c|c|c|c|c|}
\hline Conc/mM & $\begin{array}{l}s_{2} \\
\text { From (8) }\end{array}$ & $\begin{array}{l}s_{2} \\
\text { ELDOR }\end{array}$ & $\begin{array}{l}s_{\text {eff }}^{a} \\
\text { ELDOR }\end{array}$ & $\begin{array}{l}\varepsilon \\
\text { DNP }\end{array}$ & $\begin{array}{l}f \\
\text { Ref. } 21\end{array}$ & $\begin{array}{l}\xi \\
\text { Calc. }\end{array}$ \\
\hline 0.5 & - & 0.15 & $0.54 \pm 0.04$ & - & - & - \\
\hline 5 & 0.60 & 0.63 & $0.78 \pm 0.04$ & $-131 \pm 9$ & 0.78 & $0.33 \pm 0.03$ \\
\hline 10 & 0.75 & 0.78 & $0.85 \pm 0.04$ & $-157 \pm 10$ & 0.88 & $0.32 \pm 0.03$ \\
\hline 25 & 0.88 & 0.90 & $0.87 \pm 0.09$ & $-178 \pm 13$ & 0.95 & $0.33 \pm 0.04$ \\
\hline
\end{tabular}

${ }^{a}$ The values and the errors were calculated from Fig. 2 considering that $s_{1}=1$ is the upper limit and the error from ringing/noise can only reduce the total saturation. 


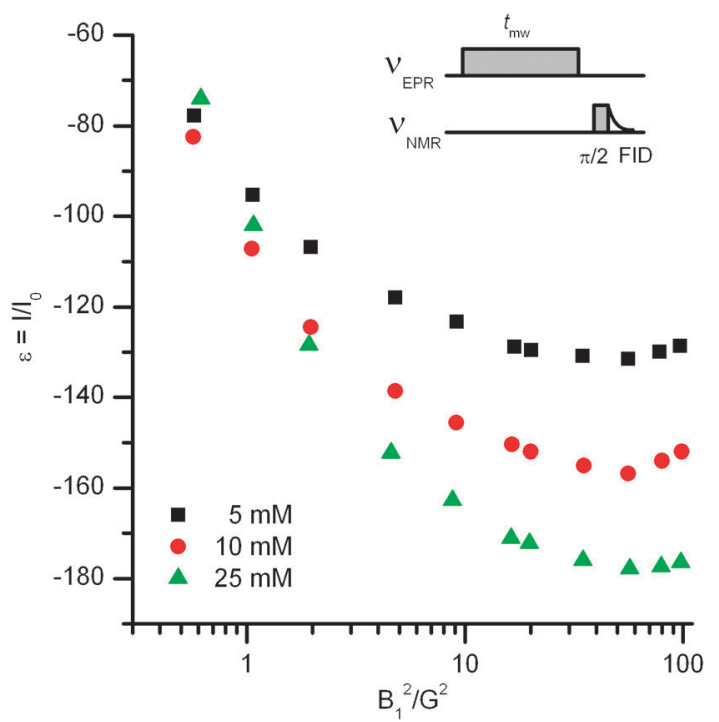

Fig. $4{ }^{1} \mathrm{H}-\mathrm{NMR}$ signal enhancement of water doped with TEMPONE-D, ${ }^{15} \mathrm{~N}$ as a function of the applied microwave $B_{1}$ field. $t_{\mathrm{mw}}$ ranges from $1 \mathrm{~s}$ to $4 \mathrm{~s}$ depending on the concentration. Experimental conditions: number of averages: 8 (DNP signal), 4096 (Boltzmann signal). Note: the slight decrease at very high $B_{1}$ fields is presumably caused by instabilities of the cavity dip due to heating of the cavity walls.

lines can be directly monitored in the ELDOR experiment even in the case in which the available microwave power does not entirely saturate the pumped EPR transition. The data also show that almost complete saturation can be achieved for a nitroxide radical dissolved in water by irradiation on one of the EPR transitions because of the saturation transfer (ELDOR) effect. Our results provide an answer to the long standing discussion about the achievable saturation factor in Overhauser DNP with nitroxide radicals.

\section{Acknowledgements}

We acknowledge financial support by the EU Design Study BIO-DNP and by the Max Planck Society. We are grateful to P. Höfer and A. Tavernier from Bruker Biospin, EPR
Division, for providing the $20 \mathrm{~W}$ CW microwave amplifier used in these experiments.

\section{Notes and references}

$\dagger$ We find that for $s_{1}=1$ and $s_{2}$ given by eqn (6) the obtained expression for $s_{\mathrm{eff}}$ is equivalent to the maximum saturation of a ${ }^{15} \mathrm{~N}$ labelled nitroxide $s_{\max }$ as derived by Armstrong and $\operatorname{Han}^{10}$ if a factor of four is considered between the definitions of the exchange rates.

1 Phys. Chem. Chem. Phys., 2010, 12, 5725-5928.

2 Appl. Magn. Reson., 2008, 34, 213-544.

3 M. Bennati, I. Tkach and M.-T. Türke, in Electron Paramagnetic Resonance, ed. B. C. Gilbert, D. M. Murphy and V. Chechik, 2011, vol. 22, RSC Publishing, DOI: 10.1039/9781849730877-00155.

4 A. W. Overhauser, Phys. Rev., 1953, 92, 411-415.

5 A. Abragam, Principles of Nuclear Magnetism, Clarendon, Oxford, 1961.

6 D. Hausser and D. Stehlik, Adv. Magn. Reson., 1968, 3, 79-139.

7 M. J. Prandolini, V. P. Denysenkov, M. Gafurov, B. Endeward and T. F. Prisner, J. Am. Chem. Soc., 2009, 131, 6090-6092.

8 P. Höfer, G. Parigi, C. Luchinat, P. Carl, G. Guthausen, M. Reese, T. Carlomagno, C. Griesinger and M. Bennati, J. Am. Chem. Soc., 2008, 130, 3254-3255.

9 R. D. Bates and W. S. Drozdoski, J. Chem. Phys., 1977, 67, 4038-4044.

10 B. D. Armstrong and S. Han, J. Chem. Phys., 2007, 127, 104508.

11 D. Sezer, M. Gafurov, M. J. Prandolini, V. P. Denysenkov and T. F. Prisner, Phys. Chem. Chem. Phys., 2009, 11, 6638-6653.

12 M.-T. Türke, I. Tkach, M. Reese, P. Höfer and M. Bennati, Phys. Chem. Chem. Phys., 2010, 12, 5893-5901.

13 R. A. Wind and J. H. Ardenkjaer-Larsen, J. Magn. Reson., 1999, 141, 347-354.

14 J. S. Hyde, J. C. W. Chen and J. H. Freed, J. Chem. Phys., 1968, 48, 4211-4226.

15 J. J. Yin, M. Pasenkiewicz-Gierula and J. S. Hyde, Proc. Natl. Acad. Sci. U. S. A., 1987, 84, 964-968.

16 J. H. Freed, J. Phys. Chem., 1967, 71, 38-51.

17 B. H. Robinson, D. A. Haas and C. Mailer, Science, 1994, 263, 490-493.

18 W. Froncisz, T. G. Camenisch, J. J. Ratke, J. R. Anderson, W. K. Subczynski, R. A. Strangeway, J. W. Sidabras and J. S. Hyde, J. Magn. Reson., 2008, 193, 297-304.

19 J. S. Hyde, J. J. Yin, W. K. Subczynski, T. G. Camenisch, J. J. Ratke and W. Froncisz, J. Phys. Chem. B, 2004, 108, 9524-9529.

20 I. V. Koptyug, S. H. Bossmann and N. J. Turro, J. Am. Chem. Soc., 1996, 118, 1435-1445.

21 M. Bennati, C. Luchinat, G. Parigi and M.-T. Türke, Phys. Chem. Chem. Phys., 2010, 12, 5902-5910.

22 D. Sezer, M. J. Prandolini and T. F. Prisner, Phys. Chem. Chem. Phys., 2009, 11, 6626-6637. 